\title{
Unravelling the complex trait of harvest index in rapeseed (Brassica napus L.) with association mapping
}

Xiang Luo, Chaozhi Ma*, Yao Yue, Kaining Hu, Yaya Li, Zhiqiang Duan, Ming Wu, Jinxing Tu, Jinxiong Shen, Bin $\mathrm{Yi}^{*}$ and Tingdong Fu

\begin{abstract}
Background: Harvest index $(\mathrm{HI})$, the ratio of grain yield to total biomass, is considered as a measure of biological success in partitioning assimilated photosynthate to the harvestable product. While crop production can be dramatically improved by increasing $\mathrm{HI}$, the underlying molecular genetic mechanism of $\mathrm{HI}$ in rapeseed remains to be shown.

Results: In this study, we examined the genetic architecture of HI using 35,791 high-throughput single nucleotide polymorphisms (SNPs) genotyped by the Illumina BrassicaSNP60 Bead Chip in an association panel with 155 accessions. Five traits including plant height (PH), branch number (BN), biomass yield per plant (BY), harvest index (HI) and seed yield per plant (SY), were phenotyped in four environments. HI was found to be strongly positively correlated with SY, but negatively or not strongly correlated with PH. Model comparisons revealed that the A-D test (ADGWAS model) could perfectly balance false positives and statistical power for $\mathrm{HI}$ and associated traits. A total of nine SNPs on the C genome were identified to be significantly associated with $\mathrm{HI}$, and five of them were identified to be simultaneously associated with $\mathrm{HI}$ and SY. These nine SNPs explained $3.42 \%$ of the phenotypic variance in $\mathrm{HI}$.

Conclusions: Our results showed that $\mathrm{HI}$ is a complex polygenic phenomenon that is strongly influenced by both environmental and genotype factors. The implications of these results are that $\mathrm{HI}$ can be increased by decreasing $\mathrm{PH}$ or reducing inefficient transport from pods to seeds in rapeseed. The results from this association mapping study can contribute to a better understanding of natural variations of $\mathrm{HI}$, and facilitate marker-based breeding for $\mathrm{HI}$.
\end{abstract}

Keywords: Harvest index, Complex traits, Brassica napus, Association mapping, Correlation

\section{Background}

Yield in rapeseed (Brassica napus L.; AACC, $2 \mathrm{n}=38$ ) has attracted the interest of plant breeders for many years. In the past decades, the productivity levels have increased due to the extensive use of heterosis in hybrid breeding. However, the average heterosis percentage for vegetative biomass, seeds per pod and 1,000-seed weight are only $25-30 \%, 17.5 \%$, and $1.8 \%$, respectively [1]. Leaves, pods, and other above-ground green tissues are able to photosynthesize as "source" organs, while seeds are storage organs that serve as the "sink" for photosynthetic products. The usual incomplete development

\footnotetext{
* Correspondence: yuanbeauty@mail.hzau.edu.cn; yibin@mail.hzau.edu.cn
National Key Laboratory of Crop Genetic Improvement, National Center of

* Correspondence: yuanbeauty@mail.hzau.edu.cn; yibin@mail.hzau.edu.cn Rapeseed Improvement in Wuhan, Huazhong Agricultural University, Wuhan 430070, P.R. China
}

filling of seeds and the low heterosis rate of seed weight suggest that when the "sources" are in surplus, the sinks are not fully filled or utilized. This could be an indication of unsmooth limitation in the "flow", resulting in lower heterosis in seed weight. Chhabra [2] and Shen et al. [3] observed that when the source and sink organs are not limiting, and the translocation of assimilates is the most critical limiting factor for seed yield in Brassica. Similar observations were reported for rice and triticale [4]. Therefore, the balance among photosynthetic "source", "flow" and "sink" is critical for yield improvement; this balance can be evaluated using harvest index $(\mathrm{HI})$ as the criterion.

The $\mathrm{HI}$ is the ratio of grain yield to total biomass (usually the total above-ground biomass). This index is considered as a measure of the biological success in partitioning 
assimilated photosynthate to the harvestable product $[5,6]$. As an integrative trait, HI was identified to be highly correlated with a number of yield-related traits in important crop species such as rice [7, 8] and sorghum [9-11]. Generally, the correlated traits are interrelated, and an increase in one component may lead to a decrease or an increase in others. Interestingly, the increase in $\mathrm{HI}$ almost fully accounted for the progressive increase in the grain yield potential of wheat, barley and rice between 1900 and 1980 [12]. Thus, a better understanding of the genetic mechanism of $\mathrm{HI}$ is crucial for interpreting agronomically important characters such as yield.

Association mapping, also called linkage disequilibrium (LD) mapping, utilizes the large number of historical recombination events that have occurred throughout the entire evolutionary history of the mapping population, allowing fine-scale QTL mapping [13, 14]. Very recently, association mapping performed with genomic, transcriptomic, epigenetic and metabolic data has provided abundant information on the genetic architecture of complex quantitative traits in a number of crop species such as maize [15-19], Arabidopsis [20], rapeseed [21-23], and rice $[24,25]$. However, the false positive rate caused by population structure is difficult to predict. Several methods have been proposed to deal with this problem. Aranzana et al. [26] found that removing the genetically distinct and phenotypically extreme accessions for flowering time could significantly reduce the false positive rate in Arabidopsis. Huang et al. [24] successfully corrected the elevated false positive rate by developing an analytical framework for haplotype-based de novo assembly of low-coverage sequencing data and identified candidate genes for 18 associated loci through detailed annotation in rice. Li et al. [21] and Cai et al. [22] controlled false associations in association mapping for seed quality and yield-related traits in rapeseed by using model comparisons (GLM, Q, PCA, K, PCA $+\mathrm{K}$ and $\mathrm{Q}+\mathrm{K}$ model). Yang et al. [27] studied the genetic architecture of 17 agronomic traits in an enlarged maize association panel by a new nonparametric model, the Anderson-Darling (A-D) test, also known as the ADGWAS model, and found that the false positives and statistical power were efficiently balanced. Additionally, joint linkage-association mapping strategies were proposed to evaluate the false association in soybean [28]. These reports suggested that association mapping will be a powerful approach for exploring the QTLs responsible for HI.

In the present study, a genome-wide association study (GWAS) of five traits (plant height (PH), seed yield per plant (SY), biomass yield per plant (BY), branch number (BN) and $\mathrm{HI}$ ) was performed with a panel of 155 accessions using 35,791 genomic SNPs from the Illumina BrassicaSNP60 Bead Chip. To control spurious associations, we analyzed the genetic population structure and familial relatedness in the GWAS population. Seven different mapping models were tested for the best fit of each trait. The chosen model was used to map markers associated with the five traits phenotyped in four environments. The objectives of the present study were to: (1) obtain a better understanding of $\mathrm{HI}$ of inbred lines; (2) examine the relationship between $\mathrm{HI}$ and other traits; (3) perform association mapping for the five traits; and (4) discuss the implications of the results of this study for further markerassisted selection breeding in B. napus.

\section{Methods}

\section{Plant materials and field experiments}

The genetic population consisted of 155 genetically diverse inbred lines (Additional file 1: Table S1). All the accessions were provided by the National Research Center of Rapeseed Engineering and Technology, Huazhong Agricultural University, Wuhan, China.

The 155 inbred lines were grown in a randomized complete block design with two replications in four different environments: Huanggang $\left(32.27^{\circ} \mathrm{N}, 114.52^{\circ} \mathrm{E}\right)$ and Xiangyang $\left(32.01^{\circ} \mathrm{N}, 112.08^{\circ} \mathrm{E}\right)$ in the $2011 / 2012$ growing season; and Wuhan $\left(29.58^{\circ} \mathrm{N}, 113.53^{\circ} \mathrm{E}\right)$ and Xiangyang $\left(32.01^{\circ} \mathrm{N}, 112.08^{\circ} \mathrm{E}\right)$ in the $2012 / 2013$ growing season. All four sites were located along the middle reaches of the Yangtze River in China. For convenience, the four sites are hereafter referred to as E1, E2, E3 and E4. A plot size of three rows (12 plants per row) was used with two replications. A spacing of $30 \times 20 \mathrm{~cm}$ between rows and between plants within a row was used at all locations. The management of the field experiments was performed in accordance with local standard practices. In each plot, PH, BN, BY and SY were measured for five representative $B$. napus plants at maturity. The HI (\%) was calculated as the ratio of SY to BY.

\section{Genotype and data analyses}

The whole population of inbred lines was genotyped using the Brassica $60 \mathrm{~K}$ Illumina Infinium SNP array by Emei Tongde Co. (Beijing) according to the manufacturer's protocol (http://www.illumina.com/technology/beadarraytechnology/infinium-hd-assay.html). The SNP data were clustered and called automatically using Illumina BeadStudio genotyping software. Those SNPs with either AA or $\mathrm{BB}$ frequency equal to zero (i.e., monomorphic), call frequency $<0.9$, or minor frequency $<0.05$ were excluded.

The data for the five traits were tested by analysis of variance (ANOVA) using SPSS version 19.0 (IBM Corp., Armonk, NY, USA).

\section{Genetic diversity and population structure analysis}

The population structure was inferred using the software package STRUCTURE v2.3.4 [29]. Five independent runs were performed with a $K$-value (the putative number of genetic groups) from 1 to 10 , with the length of burning period and the number of MCMC (Markov Chain Monte Carlo) 
replications after burning both set to 100,000 iterations under the 'admixture model'. The most likely $k$-value was determined by the $\log$ probability of data $[\operatorname{LnP}(\mathrm{D})]$ and an ad hoc statistic $\Delta \mathrm{k}$ based on the rate of change of $\operatorname{LnP}(\mathrm{D})$ between successive $\mathrm{k}$ values as described by Evanno et al. [30]. The cluster membership coefficient matrices of replicate runs from STRUCTURE were integrated to obtain the Q matrix using CLUMPP software [31] and graphically displayed using DISTRUCT software [32]. Nei's genetic distance [33] was estimated and used to construct an unrooted neighbor-joining tree representing the genome-wide relationship among the accessions. The tree was constructed using the Unweighted Pair-Group Method with Arithmetic mean (UPGMA) method with PowerMarker software. The tree was visualized using FigTree (http://tree.bio.ed.ac.uk/ software/figtree/). The genetic relatedness between individuals was estimated by principal component analysis (PCA) using NTSYSpc version 2.11 [34].

\section{Genome-wide association analysis}

The effects of population structure (Q, PC) and kinship (K) on the HI-related traits were evaluated by GWAS using seven models: (i) GLM, without controlling for Q and K; (ii) Q model, controlling for Q; (iii) PCA model, controlling for PC, with the top two principal components used as fixed effects; (iv) $\mathrm{K}$ model, controlling for $\mathrm{K}$; (v) $\mathrm{PCA}+\mathrm{K}$ model, controlling for both PC and K; (vi) Q+K model, controlling for both Q and K; and (vii) ADGWAS model, controlling for Q. The GLM, Q and PCA models were performed using a general linear model (GLM); the $\mathrm{K}, \mathrm{PCA}+\mathrm{K}$ and $\mathrm{Q}+\mathrm{K}$ models were performed using a mixed linear model (MLM) with optimum compression and population parameters previously determined (P3D) by variance component estimation in TASSEL $3.0[35,36]$. The A-D [27] test, also known as the nonparametric model or ADGWAS model, was performed using an $R$ script, ADGWAS (http://www.maizego.org/Resources.html). Statistically significant loci were identified by comparing $\mathrm{P}$ values with the Bonferroni threshold $(1 / 35791=2.79 \mathrm{E}-5)$.

\section{Phenotypic variation explained by multiple SNPs}

Stepwise regression was performed to examine the effect of multiple alleles with different functional polymorphisms on the $\mathrm{HI}$ traits, and to estimate the total variance explained $\left(R^{2}\right)$, using the $l m$ function in $R$ [37].

\section{Results}

\section{Phenotypic variations for the five traits}

Table 1 lists the details of the phenotypic variations of the five traits (PH, BN, SY, BY and $\mathrm{HI})$. The five traits were normally distributed in the population (except for BY in E2). In the four environments, HI ranged from 0.15 to 0.36 with an average of 0.20 to 0.27 . Comparatively, $\mathrm{HI}$ in E4 showed the lowest coefficient of variation $(9.71 \%)$, while
Table 1 Phenotypic variations of the five traits

\begin{tabular}{lllllll}
\hline Trait & Env. & Mean Ậ \pm SD & Range & Skew & Kurt & CV\% \\
\hline PH & E1 & $*$ & $*$ & $*$ & $*$ & $*$ \\
& E2 & $*$ & $*$ & $*$ & $*$ & $*$ \\
& E3 & $125.87 \pm 0.788$ & $97.00-156.70$ & -0.03 & 0.33 & 11.17 \\
& E4 & $128.77 \pm 0.956$ & $93.71-155.89$ & -0.48 & 0.39 & 12.41 \\
BN & E1 & $6.91 \pm 0.081$ & $4.40-9.25$ & 0.07 & -0.31 & 16.78 \\
& E2 & $6.15 \pm 0.072$ & $4.49-8.90$ & 0.70 & 0.27 & 16.61 \\
& E3 & $4.42 \pm 0.083$ & $4.20-7.80$ & 0.10 & 0.48 & 24.28 \\
& E4 & $7.56 \pm 0.106$ & $4.46-12.05$ & 0.31 & 0.56 & 19.44 \\
BY & E1 & $43.53 \pm 0.943$ & $21.63-71.74$ & 0.38 & -0.38 & 26.45 \\
& E2 & $29.79 \pm 0.642$ & $15.86-77.86$ & 1.77 & 8.38 & 26.35 \\
& E3 & $46.54 \pm 0.725$ & $22.00-71.50$ & 0.34 & -0.07 & 19.01 \\
& E4 & $41.03 \pm 0.888$ & $16.34-78.04$ & 0.48 & 0.53 & 26.41 \\
SY & E1 & $9.68 \pm 0.265$ & $4.11-18.11$ & 0.34 & -0.54 & 33.39 \\
& E2 & $8.09 \pm 0.179$ & $4.12-19.47$ & 1.08 & 1.82 & 26.95 \\
& E3 & $9.67 \pm 0.251$ & $4.85-20.79$ & 1.03 & 1.31 & 34.90 \\
& E4 & $9.84 \pm 0.236$ & $4.38-18.16$ & 0.44 & -0.15 & 29.24 \\
H1 & E1 & $0.22 \pm 0.003$ & $0.15-0.33$ & 0.43 & -0.29 & 17.18 \\
& E2 & $0.27 \pm 0.003$ & $0.17-0.34$ & -0.55 & 0.33 & 11.54 \\
& E3 & $0.20 \pm 0.004$ & $0.15-0.36$ & 1.32 & 2.94 & 20.06 \\
& E4 & $0.24 \pm 0.002$ & $0.19-0.30$ & 0.06 & -0.38 & 9.71 \\
\hline
\end{tabular}

Trait: $P H$ plant height, $B N$ branch number, $B Y$ biomass yield per plant, $S Y$ seed yield per plant, $H I$ harvest index.

Env: E1 Huanggang in 2011, E2 Xiangyang in 2011, E3 Wuhan in 2012,

E4 Xiangyang in 2012.

$S D$ : standard deviation. $C V(\%)$ : coefficient of variation.

*: data not collected.

SY in E3 had highest coefficient of variation (34.90 \%) among all five traits.

Two-factor ANOVA suggested that the differences caused by genotypes and environments were significant at the 0.05 and 0.01 levels, respectively, for all of the complex traits (Additional file 2: Table S2). There was not a strong correlation, or a negative correlation, between $\mathrm{HI}$ and $\mathrm{PH}$ $(-0.29$ at $\mathrm{p}=0.01$ level at $\mathrm{E} 3,0.01$ at $\mathrm{E} 4)$, but a strong positive $(\mathrm{P}=0.01)$ correlation between $\mathrm{HI}$ and SY $(0.34-0.83$ across the four different environments). The $\mathrm{BN}$ and $\mathrm{BY}$ were significantly positively $(\mathrm{P}=0.01)$ correlated with $\mathrm{HI}$ in E3, but the correlations were relatively weak in the other environments (Additional file 3: Table S3).

\section{Genetic diversity and population structure analysis}

The population structure of the 155 accessions was identified based on 7,600 SNPs using STRUCTURE software (Fig. 1). Clustering inference performed with possible clusters (K) from 1 to 10 showed that the most significant change of likelihood occurred when $\mathrm{K}$ increased from 2 to 3 , and the highest $\Delta \mathrm{k}$ value was observed at $\mathrm{k}=2$ (Fig. $1 \mathrm{~A}$, B, C). A radial tree created with PowerMarker had two 


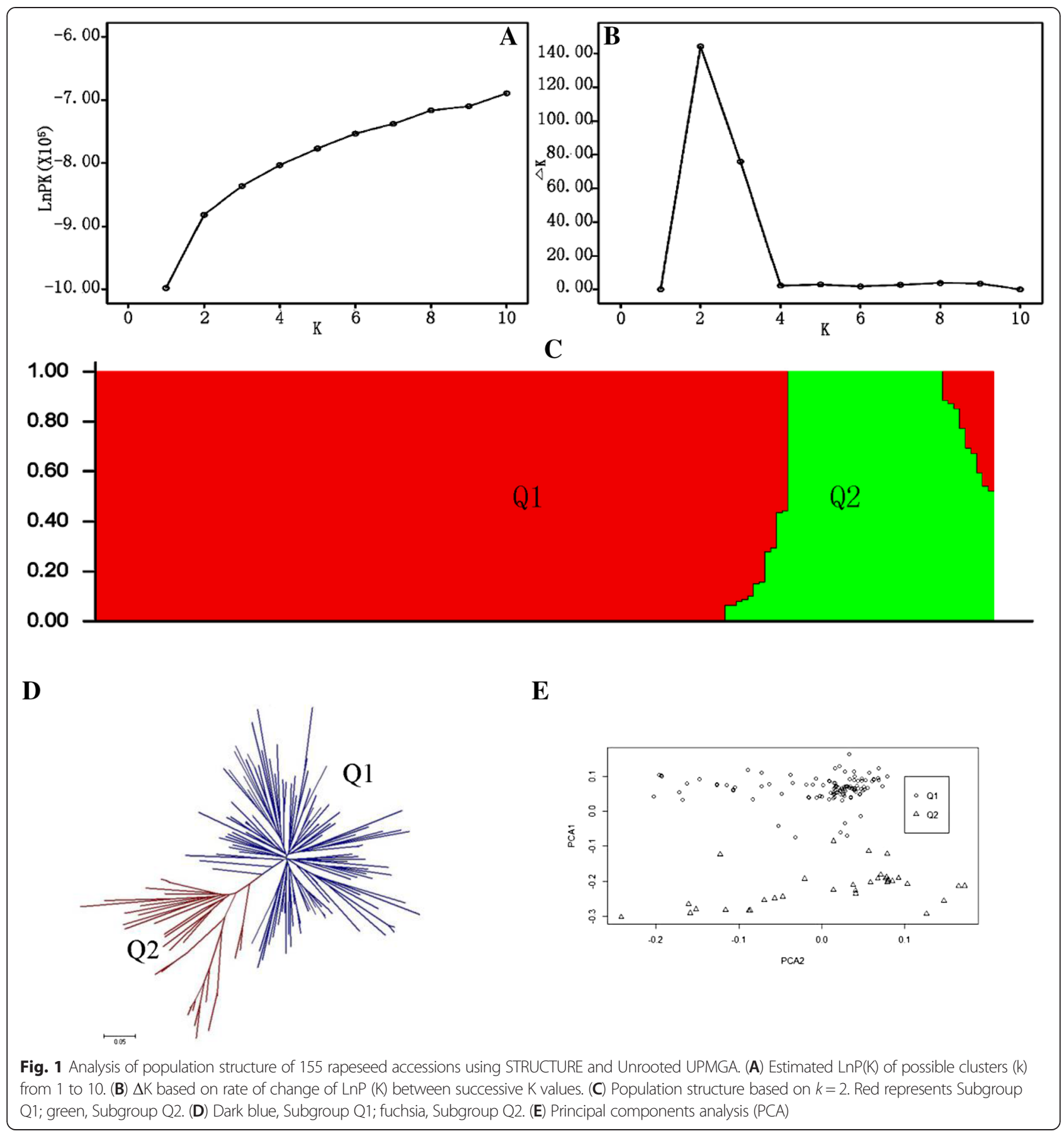

main branches for the 155 accessions (Fig. 1D). The PCA also displayed the pattern of the genetic structure of the GWAS population (Fig. 1E). All the parameters suggested that the two-group model (subgroups Q1 and Q2) sufficiently explained the genetic structure among the 155 accessions, and inspection also confirmed that the phenotypes were not randomly distributed with respect to this genetic structure. Altogether, 118 accessions belonged to subgroup Q1, and 37 accessions belonged to subgroup Q2.
Model comparisons for controlling false associations

Association analyses were performed for the five traits to evaluate the effects of population structure $(\mathrm{Q}, \mathrm{PC})$ and familial relationship (K) on controlling false associations. Among all the tested models (GLM, Q, PCA, K, $\mathrm{PCA}+\mathrm{K}, \mathrm{Q}+\mathrm{K}$ and $\mathrm{ADGWAS})$, the $\mathrm{P}$ values from the ADGWAS model showed the best fit to the expected $P$ values for all five traits (Fig. 2A, B, C, D, E). Thus, the ADGWAS model was selected to conduct association mapping for $\mathrm{HI}$ and its related traits. 


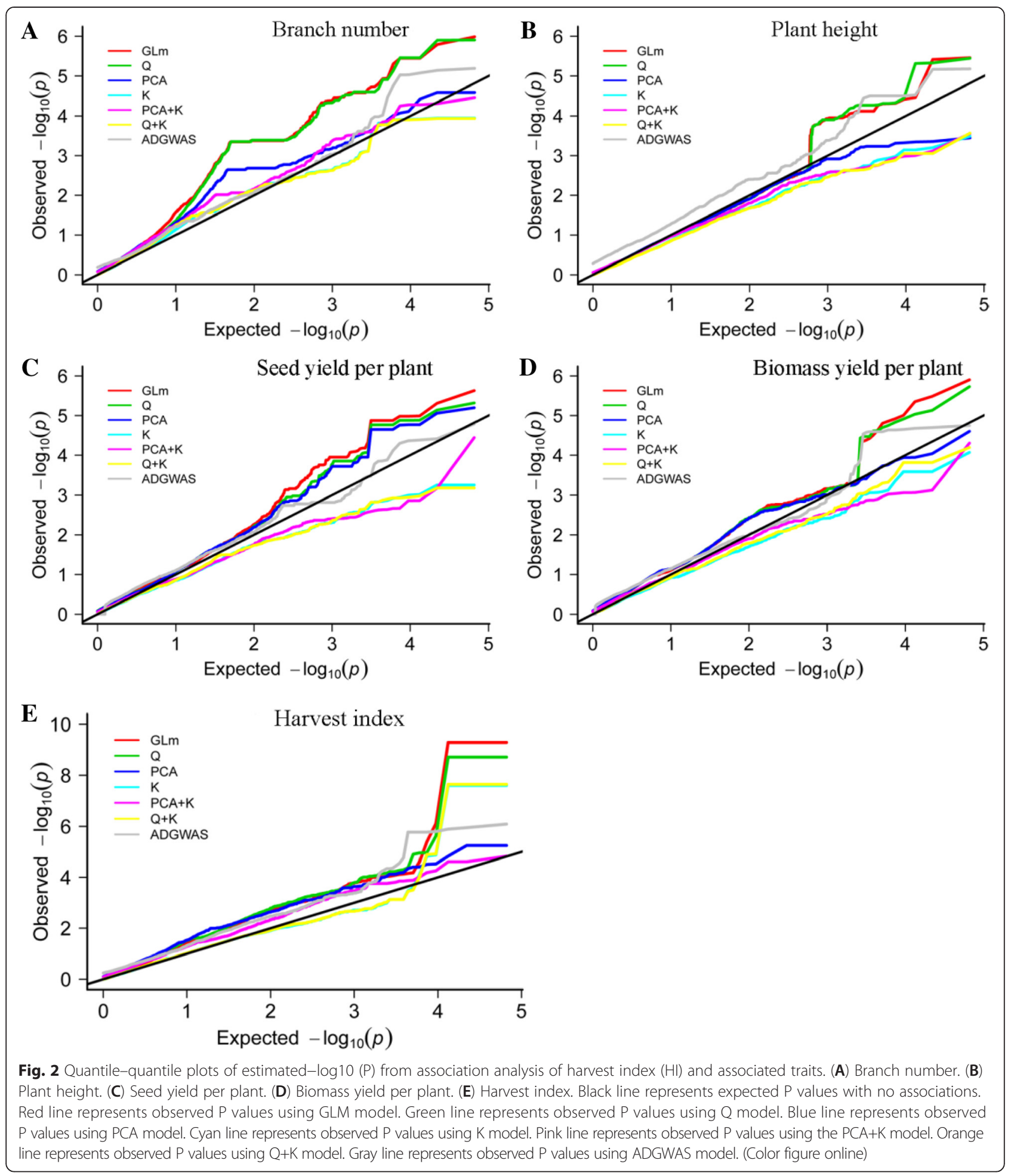

\section{Association mapping for complex traits}

In total, 35,791 polymorphisms with minor allele frequency $(\mathrm{MAF}) \geq 0.05$ were selected for association mapping. Of these, 29 SNPs were identified to be highly significantly associated with the five complex traits $(\mathrm{P}<2.79$ E-05; Table 2; Fig. 3$)$.
For PH, two SNPs (Bn-scaff_16300_1-p216539 and Bn-scaff_16300_1-p222857) were detected on C2, and these SNPs explained $1.19 \%$ of the total phenotypic variance. For $\mathrm{BN}$, six significant $\mathrm{SNPs}$ were detected on A3, and could explain $2.04 \%$ of the total phenotypic variance. For BY, only one SNP (Bn-A01-p8418102) on 
Table 2 Summary of SNPs significantly associated with complex traits

\begin{tabular}{|c|c|c|c|c|c|c|c|}
\hline Trait & SNP & Chromosome & Position & Allele & MAF & $P$ Value & $R^{2}(\%)$ \\
\hline \multirow[t]{2}{*}{$\mathrm{PH}$} & Bn-scaff_16300_1-p216539 & $\mathrm{C} 2$ & 31484854 & {$[T / C]$} & 0.22 & $6.63 \mathrm{E}-06$ & 1.19 \\
\hline & Bn-scaff_16300_1-p222857 & $\mathrm{C} 2$ & 31483855 & {$[T / G]$} & 0.23 & $6.82 \mathrm{E}-06$ & \\
\hline \multirow[t]{6}{*}{ BN } & Bn-A03-p22138059 & A3 & 22138109 & {$[T / C]$} & 0.24 & $6.45 \mathrm{E}-06$ & 2.04 \\
\hline & Bn-A03-p22145945 & A3 & 22145995 & {$[T / C]$} & 0.24 & $7.23 \mathrm{E}-06$ & \\
\hline & Bn-A03-p22237846 & $\mathrm{A} 3$ & 22237796 & {$[A / G]$} & 0.23 & $8.40 \mathrm{E}-06$ & \\
\hline & Bn-A03-p22238801 & $\mathrm{A} 3$ & 22238851 & {$[A / G]$} & 0.25 & $9.36 \mathrm{E}-06$ & \\
\hline & Bn-A03-p22149000 & $\mathrm{A} 3$ & 22148950 & {$[T / G]$} & 0.25 & $9.40 \mathrm{E}-06$ & \\
\hline & Bn-A03-p14121492 & $\mathrm{A} 3$ & 14121442 & {$[T / C]$} & 0.3 & $1.74 \mathrm{E}-05$ & \\
\hline \multirow[t]{11}{*}{ SY } & Bn-scaff_16962_1-p506943 & $\mathrm{C} 8$ & 18144556 & {$[A / G]$} & 0.34 & $1.78 \mathrm{E}-05$ & 3.96 \\
\hline & Bn-scaff_16962_1-p519147 & $\mathrm{C} 8$ & 18381806 & {$[T / C]$} & 0.34 & $2.01 \mathrm{E}-05$ & \\
\hline & Bn-scaff_16962_1-p494346 & $\mathrm{C} 8$ & 18403153 & {$[A / G]$} & 0.33 & $2.10 \mathrm{E}-05$ & \\
\hline & Bn-scaff_16962_1-p378035 & $\mathrm{C} 8$ & 18497622 & {$[T / G]$} & 0.35 & $2.24 \mathrm{E}-05$ & \\
\hline & Bn-scaff_16962_1-p504805 & $\mathrm{C} 8$ & 18395390 & {$[A / G]$} & 0.34 & $2.31 \mathrm{E}-05$ & \\
\hline & Bn-scaff_16962_1-p543498 & $\mathrm{C} 8$ & 18359355 & {$[A / G]$} & 0.33 & $2.31 \mathrm{E}-05$ & \\
\hline & Bn-scaff_16962_1-p359041 & $\mathrm{C} 8$ & 18499598 & {$[\mathrm{~T} / \mathrm{C}]$} & 0.35 & $2.55 \mathrm{E}-05$ & \\
\hline & Bn-scaff_16962_1-p376380 & $\mathrm{C} 8$ & 18498612 & {$[T / G]$} & 0.36 & $2.55 \mathrm{E}-05$ & \\
\hline & Bn-scaff_16962_1-p432767 & $\mathrm{C} 8$ & 18466493 & {$[A / G]$} & 0.36 & $2.55 \mathrm{E}-05$ & \\
\hline & Bn-scaff_16962_1-p441134 & $\mathrm{C} 8$ & 18458145 & {$[T / G]$} & 0.36 & $2.55 \mathrm{E}-05$ & \\
\hline & Bn-scaff_16962_1-p542817 & $\mathrm{C} 8$ & 18365405 & {$[A / G]$} & 0.34 & $2.66 \mathrm{E}-05$ & \\
\hline BY & Bn-A01-p8418102 & $\mathrm{A} 1$ & 8418052 & {$[A / G]$} & 0.39 & $1.63 \mathrm{E}-05$ & 0.76 \\
\hline \multirow[t]{9}{*}{$\mathrm{HI}$} & Bn-scaff_16231_1-p2306931 & $\mathrm{C} 8$ & 18546937 & {$[T / C]$} & 0.49 & $8.14 \mathrm{E}-07$ & 3.42 \\
\hline & Bn-scaff_16962_1-p426711 & $\mathrm{C} 8$ & 18472658 & {$[T / C]$} & 0.35 & $1.12 \mathrm{E}-06$ & \\
\hline & Bn-scaff_16231_1-p2303901 & $\mathrm{C} 8$ & 18301213 & {$[A / G]$} & 0.5 & $1.28 \mathrm{E}-06$ & \\
\hline & Bn-scaff_16962_1-p378035 & $\mathrm{C} 8$ & 18497622 & {$[T / G]$} & 0.35 & $1.59 \mathrm{E}-06$ & \\
\hline & Bn-scaff_16962_1-p359041 & $\mathrm{C} 8$ & 18499598 & {$[\mathrm{~T} / \mathrm{C}]$} & 0.35 & $1.68 \mathrm{E}-06$ & \\
\hline & Bn-scaff_16962_1-p376380 & $\mathrm{C} 8$ & 18498612 & {$[T / G]$} & 0.36 & $1.68 \mathrm{E}-06$ & \\
\hline & Bn-scaff_16962_1-p432767 & $\mathrm{C} 8$ & 18466493 & {$[\mathrm{~A} / \mathrm{G}]$} & 0.36 & $1.68 \mathrm{E}-06$ & \\
\hline & Bn-scaff_16962_1-p441134 & $\mathrm{C} 8$ & 18458145 & {$[T / G]$} & 0.36 & $1.68 \mathrm{E}-06$ & \\
\hline & Bn-scaff_16962_1-p351914 & C8 & 18506723 & {$[T / G]$} & 0.48 & $1.71 \mathrm{E}-05$ & \\
\hline
\end{tabular}

MAF Minor allele frequency, $R^{2}(\%)$ Amount of phenotypic variation for each trait explained by multiple SNPs.

A1 was detected $(\mathrm{P}=1.63 \mathrm{E}-05)$. This SNP could only explain $0.76 \%$ of the total phenotypic variance. For SY, 11 SNPs on C8 were detected. These SNPs could explain $3.96 \%$ of the total phenotypic variance. For HI, nine SNPs on C8 were detected. These SNPs could explain $3.42 \%$ of the total phenotypic variance. Among these SNPs, five were co-associated with HI and SY, but there were no SNPs that were co-associated with $\mathrm{HI}$ and the other three traits (PH, BN and BY). LD analyses showed that $\mathrm{r}^{2}$ values of most pairs of the multiple SNPs on C8 were $>0.20$, and those of most pairs of multiple SNPs on A3 were $>0.50$, except the BnA03-p14121492 (Additional file 4: Figure S1). These results suggested that the majority of the multiple SNPs on C8 and A3 were in high linkage disequilibrium with each other [38].

\section{Discussion}

\section{Phenotypic variations in harvest index}

For crops such as rice, wheat, barley and maize, HI has been shown to be a variable factor, with a value of approximately 0.50 [39]. Soybean, one of the most important oil crops, has a HI ranging from 0.4 to 0.6 $[40,41]$, and the HI has been successfully maximized during breeding [42]. However, the average HI of $B$. napus was reported to be approximately $0.2-0.3[43,44]$. In our study, the range of the HI of the GWAS population could be $>0.30$ in a single environment (Table 1), indicating that there is still great potential for $\mathrm{HI}$ improvement. Therefore, increasing HI might be an alternative strategy to increase seed yield gain in B. napus.

Quantitative traits related to harvest index show a range of sensitivities to environmental factors. Yang 


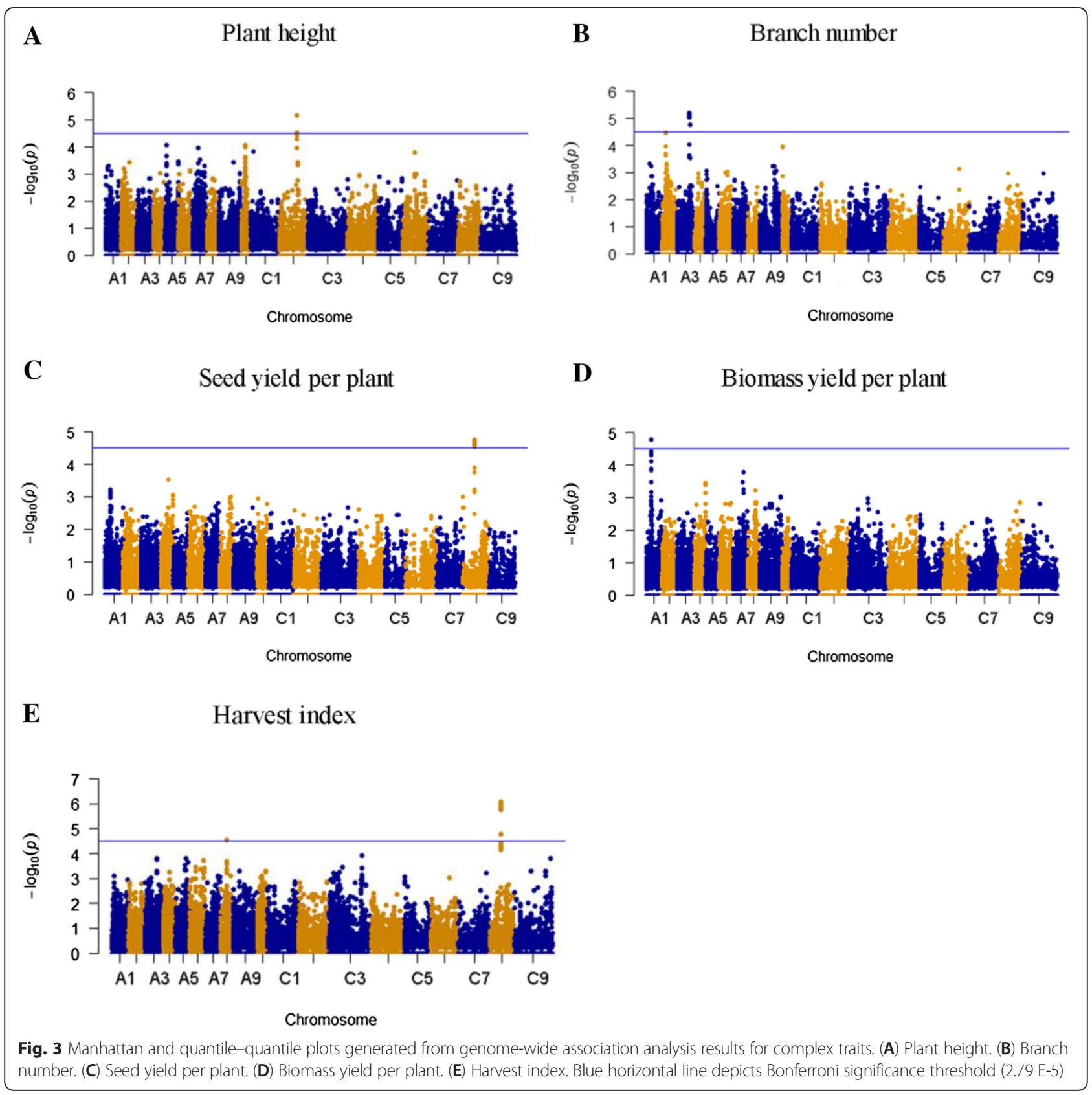

et al. [39] showed that proper crop management holds great promise to enhance the HI of rice. D'Andrea [45] evaluated the effects of the genotype and environment interaction on variations in plant grain yield, $\mathrm{HI}$ and biomass production at maturity in maize. In the present study, $\mathrm{HI}$ and its associated traits were significantly affected by the environment and genotype (Additional file 2: Table S2), consistent with the results of a study on rice [46].

The correlations among $\mathrm{HI}$ and other traits indicated that HI was negatively or not strongly positively correlated with PH (Additional file 3: Table S3). Interestingly, the HI of rice has increased primarily due to the introduction of the semi-dwarf gene [47, 48]. In this study, HI was strongly significantly correlated with SY in multiple environments, but exhibited a complex relationship with BY and BN (Additional file 3: Table S3). Rapeseed plants normally have flourishing leaves, pods, flowers and other above-ground green tissues, but their seeds are often only partially filled, probably because most of the photosynthetic products are stored in flowers and pods, rather than seeds. Also, the photosynthetic products in pods are not efficiently transported to seeds, resulting in poor seed filling. These results have 
laid the genetic basis for increasing $\mathrm{HI}$ in rapeseed by properly decreasing $\mathrm{PH}$ and/or improving transport efficiency from pods to seeds.

\section{Population structure and model comparison}

Our results demonstrate the significant potential effect of population structure on the false positive rate in association mapping. Among the seven models, the GLM model performed similarly to the $\mathrm{Q}$ model, $\mathrm{K}$ model and $\mathrm{Q}+\mathrm{K}$ model for all five traits (Fig. 2A, B, C, D, E). The PCA model, $\mathrm{PCA}+\mathrm{K}$ model, $\mathrm{K}$ model and $\mathrm{Q}+\mathrm{K}$ model performed better than the GLM model and the Q model, and might indicate potentially false negatives for $\mathrm{BN}, \mathrm{PH}$ and BY (Fig. 2A, B, D). For HI, the $\mathrm{K}$ and $\mathrm{Q}+\mathrm{K}$ models did not perform better than the GLM and Q models, and PCA, and $\mathrm{PCA}+\mathrm{K}$ models could reduce false positives, but may have indicated potentially false negatives (Fig. 2E). Compared with the other six models, ADGWAS showed the best fit for the association analysis (Fig. 2). Therefore, the efficiency of the seven models varied from trait to trait. To reduce the frequencies of false positives and false negatives, the ADGWAS model was used for the association analysis of $\mathrm{HI}$ and its related traits.

Several methods have been proposed to deal with false positives caused by population structure. Flowering time is likely involved in local adaptation, and removing the genetically distinct and phenotypically extreme accessions can indeed reduce the false positive rate [26]. However, no information is available about other traits such as yield and resistance. Haplotype-based de novo assembly of the sequencing data is an alternative approach to estimate the effect of population structure on association statistics [24]. We did not try this approach for two reasons: first, rapeseed is an allopolyploid species with a complex genome structure and a number of repeat sequences. Second, to date, there have been no reports on map-based cloning of a causal gene in a QTL of rapeseed. The combination of association mapping and linkage mapping can provide both the power and resolution needed to detect QTL of interest, and have proven to be more successful than either strategy alone [28]. Thus, the linkage mapping strategy will be used in our future work to identify the potential candidate genes by map-based cloning.

\section{Genetic dissection of harvest index}

The HI is an integrative trait including the net effects of all physiological processes during the crop cycle, and is correlated with yield-related traits. The phenotypic expression of $\mathrm{HI}$ is theoretically affected by genes responsible for yield-related traits. Li et al. [21] detected an associated SNP Bn-A10-p12639538 at 2.67 Mb of A7, which explained $4.9 \%$ of the total seed weight variation in rapeseed. Cai et al. [22] identified 43 loci $(P<0.001)$ associated with plant height, first branch height, inflorescence length, silique length, seeds per silique and seed weight in rapeseed. Li et al. [23] identified 13 consensus QTL for seed weight and 9 QTL for silique length; these QTL explained $0.7-67.1 \%$ and $2.1-54.4 \%$ of the phenotypic variance in seed weight and silique length in rapeseed, respectively. In our previous study, a functional marker derived from the sucrose transporter gene (SUT) was co-localized with a seed yield QTL in B. napus [49], and allelic variations in $B n A 7 . S U T 1$ were associated with seed yield-related traits (BnA7.SUT1.b and its promoter were linked to higher seed yield, while BnA7.SUT1.a was associated with increased seed weight) [50]. However, no QTL or loci was identified to be directly or indirectly associated with $\mathrm{HI}$ in rapeseed, and no QTL, or loci was common with the SNPs detected in the present study. To our knowledge, this is the first report of a QTL analysis of HI-related traits in rapeseed. In the present study, nine SNPs were detected to significantly associate with $\mathrm{HI}$, and could explain $3.42 \%$ of the observed variation (Table 2). These results have confirmed that $\mathrm{HI}$ is a complex polygenic phenomenon in rapeseed, like in rice [51]. Five SNPs were detected to significantly associate with both HI and SY. These SNPs might represent a shared genetic mechanism between the $\mathrm{HI}$ and SY in rapeseed. Additionally, PH was correlated with HI, but no SNP was simultaneously associated with $\mathrm{PH}$ and $\mathrm{HI}$, possibly because the SNPs associated with PH did not directly affect HI. None of the nine HI SNPs co-located with BY and BN SNPs, which was largely consistent with the observation that there was a significant phenotypic correlation between these traits only in E3, and not in the other three environments (Additional file 3: Table S3).

\section{Conclusions}

A whole genome scan identified a total of nine significant SNPs for HI. The results can contribute to a better understanding of natural variations of $\mathrm{HI}$, and provide a useful resource for marker-assisted selection breeding.

\section{Availability of supporting data}

The data sets supporting the results of this article are included within the article and its additional files.

\section{Additional files}

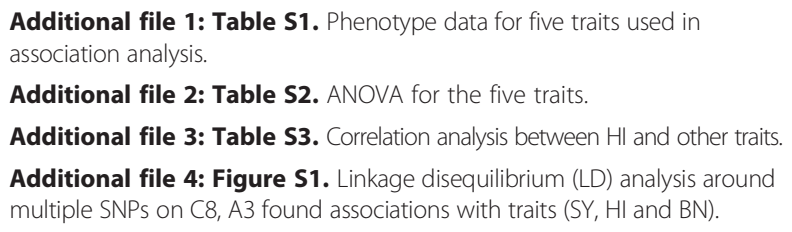

Competing interests

The authors declare that they have no competing interests. 


\section{Authors' contributions}

$\mathrm{CM}$ and $\mathrm{BY}$ designed the experiment. $\mathrm{XL}, \mathrm{YY}, \mathrm{ZD}, \mathrm{YL}$ and $\mathrm{MW}$ collected the experimental data. $X \mathrm{~L}$ and $K N$ analyzed data, and $X L$ interpreted the results and wrote the manuscript. All authors have read, edited and approved the current version of the manuscript.

\section{Acknowledgements}

This work was funded by the National Support Plan (2010BAD01B), the Central College Fund (2011PY155), and the National High Technology Research and Development Program of China (2011AA10A104, 2012AA101107).

\section{Received: 18 December 2014 Accepted: 1 May 2015} Published online: 12 May 2015

\section{References}

1. Fu TD, Zhou YM. Progress and future development of hybrid rapeseed in China. Engineering Science. 2013;5(11):13-8.

2. Chhabra ML. Translocation pattern of assimilates in India mustard. Proc 7th Int Rapeseed Cong, May 11-14, Poznan, Poland. 1987;3:804-12.

3. Shen JX, Fu TD, Yang GS. Genetic analysis of rapeseed self-incompatibility lines reveals significant heterosis of different patterns for yield and oil content traits. Plant Breed. 2005;124(2):111-6.

4. Li ZB, Xiao XH, Zhu YG. Studies and practice of Hybrid Rice. Shanghai: Shanghai Science and Technology Press; 1982 (in Chinese).

5. Li P, Zhu LH, Zhu YG. Characterization of QTLs for Harvest Index and Source-sink Characters in a DH Population of Rice (Oryza sativa L.). Acta Genetica Sinica. 2003;30(12):1118-26.

6. Sinclair TR. Historical changes in harvest index and crop nitrogen accumulation. Crop Sci. 1998;38(3):638-43.

7. Marri PR, Sarla N, Reddy LV, Siddiq EA. Identification and mapping of yield and yield related QTLs from an Indian accession of Oryza rufipogon. BMC Genet. 2005;6(1):33.

8. Sabouri H, Sabouri A, Dadras AR. Genetic dissection of biomass production harvest index and panicle characteristics in indica-indica crosses of Iranian rice (Oryza sativa L.) cultivars. Aust J Crop Sci. 2009;3(3):155-66.

9. Can ND, Yoshida T. Genotypic and phenotypic variances and covariances in early maturing grain sorghum in a double cropping. Plant Prod Sci. 1999;2(1):67-70

10. Mohammad D, Cox PB, Posler GL, Kirkham MB, Hussain A, Khan S. Correlation of characters contributing to grain and forage yield and forage quality in sorghum (sorghum bicolor). Indian J Agr Sci. 1993;63:92-5

11. Soltani A, Rezai AM, Khajeh Pour MR. Genetic variability for some physiological and agronomic traits in grain Sorghum (Sorghum bicolor L.). JWSS-Isfahan University of Technology. 2001;5(1):127-37.

12. Hay RKM. Harvest index: a review of its use in plant breeding and crop physiology. Ann Appl Biol. 1995;126(1):197-216.

13. Nordborg M, Weigel D. Next-generation genetics in plants. Nature. 2008;456:720-3

14. Nordborg M, Tavare S. Linkage disequilibrium: what history has to tell us. Trends Genet. 2002;18:83-90

15. Tian F, Bradbury PJ, Brown PJ. Genome-wide association study of maize identifies genes affecting leaf architecture. Nat Genet. 2011;43:159-62.

16. Kump KL, Bradbury PJ, Wisser RJ. Genome-wide association study of quantitative resistance to southern leaf blight in the maize nested association mapping population. Nat Genet. 2011;43(2):163-8.

17. Poland JA, Bradbury PJ, Buckler ES. Genome-wide nested association mapping of quantitative resistance to northern leaf blight in maize. Proc Natl Acad Sci. 2011;108(17):6893-8

18. Li H, Peng Z, Yang X. Genome-wide association study dissects the genetic architecture of oil biosynthesis in maize kernels. Nat Genet. 2013;45(1):43-50.

19. Chen ZJ. Genomic and epigenetic insights into the molecular bases of heterosis. Nat Rev Genet. 2013;14:471-82.

20. Atwell S, Huang YS, Vilhjálmsson BJ. Genome-wide association study of 107 phenotypes in Arabidopsis thaliana inbred lines. Nature. 2010;465(7298):627-31.

21. Li F, Chen B, Xu K, Wu J, Song W, Bancroft I, Harper AL, Trick M, Liu S, Gao G, Wang N, Yan G, Qiao J, Li J, Li H, Xiao X, Zhang T, Wu X. Genome-wide association study dissects the genetic architecture of seed weight and seed quality in rapeseed (Brassica napus L.). DNA research. 2014;21(4):355-67.
22. Cai $D$, Xiao $Y$, Yang $W$. Association mapping of six yield-related traits in rapeseed (Brassica napus L.). Theor Appl Genet. 2014;127(1):85-96.

23. Li N, Shi J, Wang X. A combined linkage and regional association mapping validation and fine mapping of two major pleiotropic QTLs for seed weight and silique length in rapeseed (Brassica napus L.). BMC Plant Biol. 2014;14(1):114.

24. Huang X, Wei X, Sang T. Genome-wide association studies of 14 agronomic traits in rice landraces. Nat Genet. 2010;42(11):961-7.

25. Chen W, Gao Y, Xie W. Genome-wide association analyses provide genetic and biochemical insights into natural variation in rice metabolism. Nat Genet. 2014;46(7):714-21.

26. Aranzana MJ, Kim S, Zhao K. Genome-wide association mapping in Arabidopsis identifies previously known flowering time and pathogen resistance genes. PLoS Genet. 2005;1(5), e60.

27. Yang N, Lu Y, Yang X, Huang J, Zhou Y. Genome wide association studies using a new nonparametric model reveal the genetic architecture of 17 agronomic traits in an enlarged maize association panel. PLoS Genet. 2014;10(9), e1004573

28. Korir PC, Zhang J, Wu K, Zhao T, Gai J. Association mapping combined with linkage analysis for aluminum tolerance among soybean cultivars released in Yellow and Changjiang River Valleys in China. Theor Appl Genet. 2013;126(6):1659-75.

29. Pritchard JK, Stephens M, Donnelly P. Inference of population structure using multilocus genotype data. Genetics. 2000;155(2):945-59.

30. Evanno G, Regnaut S, Goudet J. Detecting the number of clusters of individuals using the software STRUCTURE: a simulation study. Mol Ecol. 2005;14(8):2611-20.

31. Jakobsson M, Rosenberg NA. CLUMPP: a cluster matching and permutation program for dealing with label switching and multimodality in analysis of population structure. Bioinformatics. 2007:23(14):1801-4.

32. Rosenberg N. DISTRUCT: a program for the graphical display of population structure. Mol Ecol Notes. 2004:4:137-8.

33. Nei M, Takezaki N. Estimation of Genetic Distances and Phylogenetic Trees from DNA Analysis. In: Proceedings of the Fifth World Congress on Genetics Applied to Livestock Production. Guelph, Ontario, Canada: University of Guelph; 1983. p. 405-12.

34. Rohlf FJ. Multivariate Analysis System, Version 2.10 e. Applied Biostatistics. 2000; Inc, New York.

35. Yu J, Pressoir G, Briggs WH. A unified mixedmodel method for association mapping that accounts for multiple levels of relatedness. Nat Genet. 2006:38:203-8.

36. Yang X, Yan J, Shah T. Genetic analysis and characterization of a new maize association mapping panel for quantitative trait loci dissection. Theor Appl Genet. 2010;121:417-31.

37. Ihaka R, Gentleman R. R: a language for data analysis and graphics. J Comput Graph Stat. 1996;5(3):299-314.

38. Hasan M, Friedt W, Pons-Kühnemann J, Freitag NM, Link K, Snowdon RJ. Association of gene-linked SSR markers to seed glucosinolate content in oilseed rape (Brassica napus ssp. napus). Theor Appl Genet. 2008;116(8):1035-49.

39. Yang J, Zhang J. Crop management techniques to enhance harvest index in rice. J Exp Bot. 2010;61:3177-89.

40. Board JE, Modali H. Dry matter accumulation predictors for optimal yield in soybean. Crop Sci. 2005;45(5):1790-9.

41. Yazdani F, Allahdadi I, Akbari GA. Impact of superabsorbent polymer on yield and growth analysis of soybean (Glycine max L.) under drought stress condition. Pak J Biol Sci. 2007;10(23):4190-6.

42. Zhu XG, Long SP, Ort DR. Improving photosynthetic efficiency for greate yield. Annu Rev Plant Biol. 2010;61:235-61.

43. Yuan W, Guan C. Harvest index in rapeseed affected by a few physiological factors. Zuo Wu Xue Bao. 1996;23(5):580-6

44. Yuan WZ, Guan CY, Liao AL. Contribution of harvest index to seed yield of rapeseed. J Nat Hunan Norm Univ. 1999;22(1):65-9.

45. D'Andrea KE, Otegui ME, de la Vega AJ. Multi-attribute responses of maize inbred lines across managed environments. Euphytica. 2008;162(3):381-94

46. Li X, Yan W, Agrama H. Unraveling the complex trait of harvest index with association mapping in rice (Oryza sativa L.). PLoS ONE. 2012;7(1):e29350.

47. Peng S, Cassman KG, Virmani SS. Yield potential trends of tropical rice since the release of IR8 and the challenge of increasing rice yield potential. 1999. p. 1552-9 
48. Zhang Q. Strategies for developing green super rice. Proc Natl Acad Sci. 2007;104(42):16402-9.

49. Li YY, Shen JX, Wang TH, Chen QF, Zhang XG, Fu TD, et al. QTL analysis of yield-related traits and their association with functional markers in Brassica napus.L. Aust J Agr Res. 2007;58(8):759-66.

50. Li F, Ma C, Wang X. Characterization of sucrose transporter alleles and their association with seed yield-related traits in Brassica napus L. BMC Plant Biol. 2011;11(1):168

51. Qu Z, Li L, Luo J. QTL mapping of combining ability and heterosis of agronomic traits in rice backcross recombinant inbred lines and hybrid crosses. PLoS ONE. 2012;7(1), e28463.

Submit your next manuscript to BioMed Central and take full advantage of:

- Convenient online submission

- Thorough peer review

- No space constraints or color figure charges

- Immediate publication on acceptance

- Inclusion in PubMed, CAS, Scopus and Google Scholar

- Research which is freely available for redistribution 